\title{
Stem cells and skin regeneration
}

\author{
Marzena Staniszewska $^{1}$, Sylwia Słuczanowska-Głąbowska ${ }^{1}$, Justyna Drukała ${ }^{2}$ \\ ${ }^{1}$ Department of Physiology, Pomeranian Medical University, Szczecin, Poland \\ ${ }^{2}$ Laboratory of Cell and Tissue Engineering, Department of Cell Biology, Faculty of Biochemistry, \\ Biophysics and Biotechnology, Jagiellonian University, Krakow, Poland
}

\begin{abstract}
Stem cells represent a great hope for regenerative medicine. In adult life, stem cell deposits are kept in organ niches; the need for tissue or organ regeneration mobilizes stem cells via the SDF-1-CXCR4 regulation axis. Constant regeneration of the skin is achieved due to stem cell differentiation within the epidermis and the hair follicle; thus, skin may serve as an excellent source of stem cells. This is of paramount importance in the treatment of chronic skin wounds and burns. (Folia Histochemica et Cytobiologica 2011; Vol. 49, No. 3, pp. 375-380)
\end{abstract}

Key words: stem cells, epidermal stem cells, VSELs, CXCR4 receptors, wound

\section{Introduction}

The great interest in the biology of stem cells (SC) is related to their capacity for self-renewal, replication and differentiation to other cells that build different tissues and organs. SCs replenish lost cells throughout an organism's lifespan. SCs have the capacity for unlimited replication that gives a population of 'sister' SCs. These cells are responsible for self-renewal and differentiate into tissue-specific cells. This process maintains the constant number of aging somatic cells, which become apoptotic. In the future, SCs could be used in the treatment and regeneration of organs and tissues. The implantation of SCs could be applied instead of the transplantation of tissue and organs. This would be a huge step in regenerative medicine [1-6].

There are several types of SCs, which differ one from another in their proliferation and differentiation capacity. The less mature SCs have greater possibilities of differentiation and replication. Previous research suggested that tissue-commited stem cells (TCSCs) showed plasticity i.e. the possibility of these cells transdifferentiating into other TCSCs under the

Correspondence address: M. Staniszewska, Department of Physiology, Pomeranian Medical University, Al. Powstancow Wielkopolskich 72, 70-111 Szczecin, Poland; e-mail: stanma@sci.pam.szczecin.pl control of environmental factors. For example, hematopoietic stem cells (HSCs) could differentiate into heart stem cells, hepatic stem cells or pancreas stem cells. The new hypothesis on this subject is that stem cell niches are not only colonized by TCSCs but also contain pluripotent stem cells (PSCs), which can differentiate into specific tissue $[7,8]$.

PSCs express embryonic markers such as Oct4, Nanong and Rex-1 and give rise to SCs specific for various tissues and organs; some of them are deposited during embryogenesis in organs and can survive in these localizations to adulthood [9-15].

Kucia and Ratajczak $[16,17]$ confirmed that bone marrow (BM) and other tissue of adults is equipped with PSCs - the very small embryonic-like stem cells (VSELs). The morphology of these cells and their immunohistochemical features are similar to those of early embryonic SCs. They were initially isolated from murine $\mathrm{BM}$ as a homogenic lineage Sca- $1^{+}$lin-CD45- which demonstrates coexpression of PSCs markers such as SSEA-1, Oct-4, Nanog and Rex-1 [18]. Direct electron microscopy showed cells with a large nucleus with euchromatin and narrow cytoplasm. The cells are isolated from human BM, circulating blood and umbilical cord blood as CD $34{ }^{+} \mathrm{CD} 1333^{+} \mathrm{CXCR} 4{ }^{+}$lin-CD $45^{-}$, small size cells ( $7 \mu \mathrm{m}$ in diameter ) [19-21]. In vitro, they differentiate into all three germ layers [16]. These cells are enriched for mRNA for skin epidermis like Trp63, Krt2-5, BNC [16]. During ontogenesis, VSELs are deposited in BM 
and in other organs (tissues) and are mobilized in cases of organ and tissue damage for their regeneration $[22,23]$. Their number is higher in young individuals and decreases with age $[7,16]$.

The stem cells migration, which is the key process in their development and regeneration, is regulated by the axis CXCR4-SDF-1 [24-26]. CXCR4 receptor has been described in many types of tissue-specific SCs including nervous tissue, skeletal muscles, heart, liver, endothelium, tubules of nephron, pigment cells of retina and embryonic PSC. SCs follow the SDF-1 gradient. The SDF-1 is expressed in stromal, endothelial, cardiac, skeletal muscle, liver, brain and renal cells. Recently, the alternative receptor for SDF-1, CXCR7, was described [26, 27]. The damage of tissue increases expression of SDF-1 that attracts $\mathrm{CXCR} 4^{+} \mathrm{SCs}$, which are necessary for organ reparation. The expression of SDF-1 can be up-regulated by HIF- $1 \alpha$ and down-regulated by steroids, granulocytes colony stimulating factor (G-CSF) and transforming growth factor (TGF- $\beta 1$ ) [25].

Investigators have found SCs in the niches of the epidermis $[9,10,13]$. PSCs as Oct $4^{+}$embryonic cells or as non-epidermal non-melanocyte $\mathrm{Oct}^{+}{ }^{+} \mathrm{Nanog}^{+}$ cells have been identified in the same niche [28]. The association of these cells with VSELs requires further investigation.

Dyce et al. [29] determined that SCs isolated from the skin include a population capable of differentiating into oocyte-like cells expressing Oct4 and other markers characteristic for oocytes. The hypothesis on the migration of cells of epiblast - primordial germ cells (PGCs) - into nongonadal niches during early embryogenesis may explain their presence in the skin.

Obtaining PSCs from the skin may potentially give us new uses for these cells in terms of treatment, and become a new experimental model for in vitro studies.

\section{Epidermal stem cells}

Keratinization and exfoliation are natural features of the epidermis. The epidermis is the tissue that undergoes continuous renewal. The process depends on the subpopulation of SCs and occurs in the stratum basale of the epidermis. In a normal epidermis, SCs constitute approximately $1-10 \%$ of the stratum basale. It is believed that the skin is the human organ tissue richest in SCs [9].

SCs of the epidermis undergo asymmetric divisions. The effect is self-renewal of epidermal stem cells (ESCs) and the development of sister cells transient amplifying cells (TACs), which undergo a limited number of mitotic divisions and ultimately differentiate. The process is very fast in vitro (Figure 1).

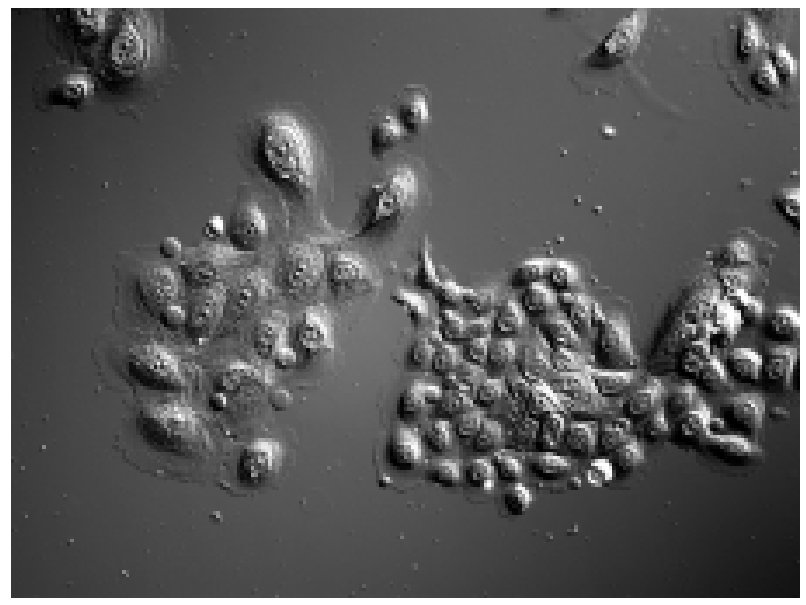

Figure 1. Secondary culture of human keratinocytes isolated from skin. Two types of colonies formed by differentiated large cells and by small progenitor epidermal cells are visualized with a Leica DM IRE2 microscope equipped with Nomarski interference contrast optics (own studies)

It is difficult to distinguish the two types of cells. It seems identification of CD71 receptor that is expressed on TACs and is lacking in tissue directed ESCs may be helpful [30]. Similarly, identification of CD90 antigen is useful to define the keratinocytes population that is enriched in SCs [31].

ESCs are not an homogenous population, and differ with homing site. They may be found in interfollicular parts of the epidermis, in the region of the outer root sheath of the hair follicle (bulge), and in the germinal matrix of the hair follicle [32]. The interfollicular SCs closely adhere to the basal lamina of the epidermis. They resemble somatic SCs. Slow cell cycle of these cells prevents the accumulation of mutations, long life span and proliferation ability provides maintenance and repair of the tissue they reside. The final differentiation into keratinocytes begins after loss of contact with the basement membrane and is related to renewal of the epidermis [9].

The hair follicle outer root sheath (bulge) subpopulation of ESCs is a source of keratinocytes and hair follicle cells. It is the large population of SCs capable of regenerating a number of skin structures including the hair follicles and sebaceous glands $[2,14,33]$. Yu et al. [28] showed in a hair follicle a population of SCs which express Oct4 and Nanog markers but are neither ESCs nor melanocyte SCs. These were probably VSELs. Thus, the epidermis may contain a heterogenic population of SCs capable of regenerating not only the epidermis.

The SCs of the bulge region have a high proliferation potential. In the epidermis, they remain quiescent because of their long cell cycle. In vitro, they 
maintain their capacity to differentiate. The hair follicle SCs generate 'daughter' cells which migrate up or down the hair follicle. In the upper part of the hair follicle, they become progenitor cells for the reconstitution of the epidermis. In the lower part of the hair follicle, they change into matrix cells. The interfollicular SCs express CD34 marker which is not expressed by bulge SCs [9, 13-15].

The cells of the bulge region have a higher potential for regeneration, a longer cell cycle and a higher clonogenic potential than interfollicular stem cells whose niche is not clearly defined [9]. Populations of bulge SCs differentiate into keratinocytes, melanocytes, smooth muscles and neurons depending on the tissue-specific inducing medium. They express embryonic markers including Oct4 and Nanog [28].

Culture of epidermal stratum basale cells results in the formation of clones described as holoclones, paraclones and meroclones. Holoclones are formed by SCs and paraclones by TACs. Meroclones contain a transitional cell type understood to be a reservoir of TACs. The differentiation of cells occurs from holoclones, through meroclones, to paraclones [32].

ESCs express $\beta 1$ and $\alpha 6$ integrins, transcription factor p63 and delta-1, and high expression of cytoplasmatic $\beta$-catenin with cellular membrane cadherins and preferentially express the cytokeratins K15 and $\mathrm{K} 19$ [14, 32]. Yu et al. [28] revealed the presence of cytokeratin $\mathrm{K} 15$ in the bulge region and expression of Nanog and Oct 4 pluripotency markers in the same localization. This showed that Oct $4^{+}$and $\mathrm{Nanog}^{+}$cells reside in bulges. The abovementioned cells, when raised from hair follicles, can be important sources of autologous SCs for tissue regeneration.

It has been demonstrated that $\beta 1$ and $\alpha 6$ integrins are present in the stratum basale cells of the epidermis. Migration from this area toward the skin surface results in a decreased expression of $\beta 1$ integrin. The cells with high expression of this integrin are mainly SCs, which are able to cover for basis of collagen IV and laminin. Similarly p63, a factor that undergoes expression in the nucleus, indicates those cells able to replicate and proliferate. Expression of p63 sharply decreases during differentiation.

In conclusion, $\beta 1$ integrin, $\mathrm{p} 63$ factor and the other mentioned factors can be useful as epidermis SCs high markers. Abundant melanocytes can be removed from culture by marker c-kit. Additionaly it is possible to use metabolic dye 123 rhodamine for identification of ESCs. Sorting of cells Rh 123 (-) and $\beta 1$-integrin (+) enable isolation of enriched SCs fraction forming holoclones in culture [32, 34].

The proliferation of ESCs depends on growth factors produced by skin fibroblasts including fibroblast growth factor (FGF-4), keratinocytes growth factor (KGF), epidermal growth factor (EGF), hepatocyte growth factor (HGF) and IL-6 and onkostatin M $[9,14,15,32]$.

\section{Application of epidermal stem cells}

ESCs open a new perspective on the healing of different types of skin disorder including severe burns, chronic leg ulcers, skin cancer, alopecia, and acne $[2,3,6,9,35]$.

The epidermis and hair follicle are distinct lineage compartments maintained by independent SCs populations. Both epidermal and follicular keratinocytes are recruited to participate in epidermal repair in response to injury. For instance, in superficial burns with destroyed interfollicular epidermis and intact hair follicles, the epidermis is rebuilt because of SCs present in bulges. It is believed that these cells participate only transiently in the initial resurfacing of the wound and are replaced by SCs from the original epidermal compartment. However, their daughter cells have been found for months after a wound has healed [12]. When hair follicles are destroyed due to deep burns, the epidermis can regrow only via cells migrating from the edges of intact skin [36].

Skin regeneration in chronic wounds is similar. In intact skin, cells do not move. They migrate vertically in the epidermis, being passively pushed by cells growing at the stratum basale and gradually differentiating to keratinocytes. In injured skin, they exhibit lateral migration and during wound healing (epithelial) cells migrate laterally to cover the wound surface [9].

Limat et al. [6] collected and cultured keratinocytes expanded from the outer root sheaths of hair follicles. Cells were organized in epidermal equivalent prepared from autologous keratinocytes and applied to the surface of a recurrent leg ulcer. The larger the area of implantation, the faster the re-epithelialization occurred. Within eight weeks, one third of the ulcers had healed. Patients reported reduced pain and no side effects of this treatment.

Drukała et al. [3] evaluated the migration of human keratinocytes from primary and secondary cells culture on polystyrene, collagen, and fibrin glue. Results showed furthest displacement on polystyrene, followed by fibrin glue and collagen, but the fastest was on the fibrin glue surface. In comparison, these parameters were much worse when keratinocytes were organized in sheets. Furthermore, five days after transplantation of single-cell suspension of autologous keratinocytes to patients with chronic leg ulcers, the formation of proliferating cell islands was observed. In addition, pain was relieved significantly and wound 
secretion was reduced. Results suggest that multifocal skin grafting, as well as the transplantation of single-cell suspension, can be very effective treatments for long-lasting leg ulcers. This effect is absent from the transplantation of large skin sheets because locomotion concerns only edge cells. In addition, in vivo wound healing that requires fibroblast growth and collagen synthesis can be stimulated in the presence of fibrin glue (Figure 2).

Bone marrow SCs and other tissue niches have been identified as sources of cells which can participate in the regeneration of injured tissues and organs $[18,22,23]$. Their mobilization is dependent on the secretion of tissue factors. The CXCR4-SDF-1 axis is the most important regulator of migration. It provides SCs to participate in the rebuilding of damaged organs thanks to attraction by secreted chemotactic substances [26].

Bone marrow derivative cells (BMDCs) are not detected in steady-state epidermal homeostasis. Fan et al. [37] indicated that in a skin wound-healing model, the epidermis contains only rare bone marrow-derived keratinocytes. This may suggest that BMDCs are not required for keratinocytes repopulation. Borue et al. [38], unlike above-mentioned studies, found that skin damage may affect the degree of engraftment of BMDCs to the skin. Probably, BMDCs do not engraft as ESC, but assist in early wound healing by engrafting as TACs and differentiate into keratinocytes. Lethal irradiation and reconstitution of BM indicate engraftment to the wound skin of transplanted BMDCs. Expression of Ki 67, a marker for actively cycling cells, correlates with the increased number of donor-derived cells within the wound. Donor-derived cytokeratin 5-expressing cells were rare, suggesting that BMDCs do not engraft as ESCs, and the level of them peaked and decreased over time, further suggesting that BMDCs may assist in early wound healing by engrafting as TACs, which then differentiate into keratinocytes.

According to Inokuma et al. [39], the major regulator of migration of BMDCs into skin is CTACK. It is constitutively expressed in normal skin and upregulated in wounds. Furthermore, CD $34^{+}$bone marrow cells expressed CCR 10, the ligand for CTACK.

The bone marrow-derived keratinocytes may build a provisional epidermal layer and later be replaced by keratinocytes migrating from surrounding skin. Probably, after injury, BMDCs are recruited by pro-inflammatory cytokines. Their homing to the skin is mediated by CTACK secreted by keratinocytes. In chronic wounds, the recruitment of BMDCs seems to be impaired by blocking of CTACK mediated axis.
Inhibition mediated by PSGL-1 released from neutrophils present in the inflammatory center may delay the healing of chronic wounds and confirm previous suggestions about this chemotactic way [40].

Furthermore, direct transplantation of bone marrow cells on damaged skin starts their differentiation into non-hematopoietic skin structures. According to Krause et al. [5], an enriched population of HSC that homes to bone marrow can be engrafted to peripheral tissues. Thus, rare cells homing to bone marrow can create a long-term population of SCs with high differentiation capacity, even into skin cells. The connection of these findings with presence of VSELs in bone marrow niche should be researched.

Badiavas et al. [1] indicated that direct application of autologous bone marrow-derived cells can lead to closure of non-healing chronic wounds and dermal rebuilding. These results suggested engraftment of applied cells, which was confirmed in biopsy.

Kataoka et al. [4] transplanted mouse bone marrow cells onto damaged skin. They observed reconstitution of fully differentiated skin within three weeks. Donor cells were found in the epidermis, hair follicles, sebaceous glands, and dermis. The localization and morphology of those cells, immunohistochemistry, and specific staining confirmed that the bone marrow cells had differentiated into epidermal keratinocytes, sebaceous gland cells and follicular epithelial cells.

Since the SDF-1 - CXCR4 axis has been recognized as an important regulator of trafficking of SCs, it has become a molecular target for various therapeutic interventions. It has been demonstrated that the expression of CXCR4 could be inhibited at the transcriptional level by targeting HIF- $1 \alpha$ by chetomin, at the translation level by siRNA or antisense oligodeoxynucleotides, and at the protein receptor level by T140 or AMD 3100. Blocking the formation of lipid rafts in individuals depleted of cholesterol decreases the responsiveness of cells to an SDF-1 gradient. Interestingly, primes of genes coding for receptor CXCR4 is similar to binding place for HIF- $1 \alpha$. Inflammation and tissue injury result in chemotactic attraction of stem cells for regeneration [25].

The large amount of easily available epidermal stem cells enriched in early PSCs (with marker Oct-4) raises hopes of a clinical use for treating not only skin injuries but also for organ regeneration. Our unpublished data showed that burns trigger the mobilization of VSELs and progenitor cells expressing early epidermal markers into the peripheral blood. It is still too early to determine the potential role of these cells in skin regeneration. To answer this question, the optimization of VSELs expansion ex vivo and also their differentiation into keratinocytes is required. 

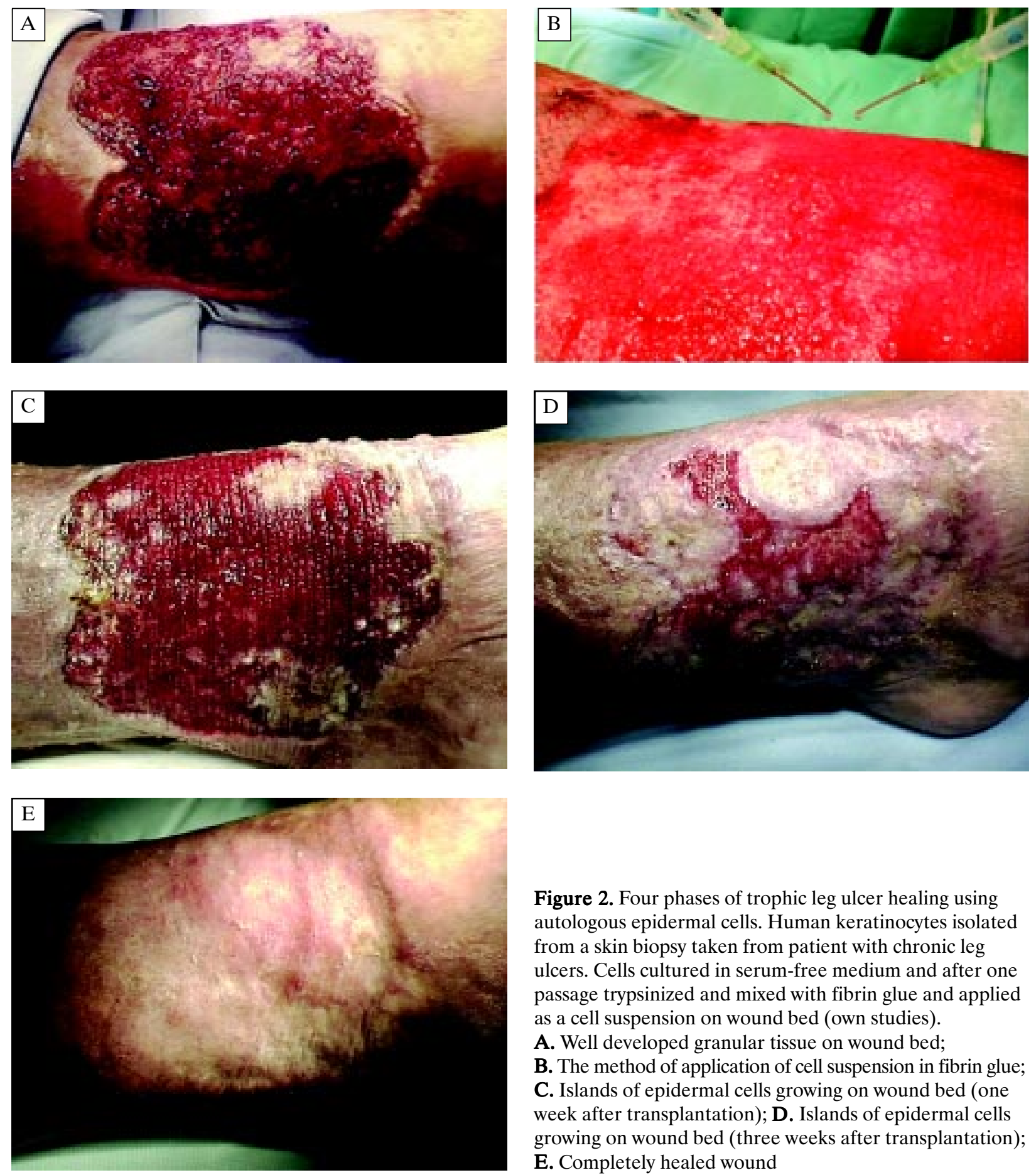

Figure 2. Four phases of trophic leg ulcer healing using autologous epidermal cells. Human keratinocytes isolated from a skin biopsy taken from patient with chronic leg ulcers. Cells cultured in serum-free medium and after one passage trypsinized and mixed with fibrin glue and applied as a cell suspension on wound bed (own studies).

A. Well developed granular tissue on wound bed;

B. The method of application of cell suspension in fibrin glue; C. Islands of epidermal cells growing on wound bed (one week after transplantation); D. Islands of epidermal cells growing on wound bed (three weeks after transplantation); E. Completely healed wound

The coming years will show if these cells can be used in regenerative medicine.

\section{Acknowledgements}

This work is supported by European Union structural founds Innovative Economy Operational Program POIG.01.01.01-00-109/09-01 and a grant from the Polish Ministry of Science and Higher Education 0619/B/P01/2009/36.

\section{References}

1. Badiavas EV, Falanga V. Treatment of chronic wounds with bone marrow-derived cells. Arch Dermatol. 2003;139: 510-516.

2. Cha J, Falanga V. Stem cells in cutaneous wound healing. Clin Dermatol. 2007;25:73-78.

3. Drukała J, Bandura L, Cieślik K, Korohoda W. Locomotion of human skin keratinocytes on polystyrena, fibrin, and collagen substrata and its modification by cell-to-cell contacts. Cell Transplant. 2001;10:765-771. 
4. Kataoka K, Medina RJ, Kageyama T et al. Participation of adult mouse bone marrow cells in reconstitution of skin. Am J Pathol. 2003;163:1227-1231.

5. Krause DS, Theise ND, Collector MI et al. Multi-organ, multi-lineage engraftment by a single bone marrow-derived stem cell. Cell. 2001;105:367-377.

6. Limat A, French LE, Blal L, Saurat JH, Hunziker T, Salomon D. Organotypic cultures of autologous hair follicle keratinocytes for the treatment of recurrent leg ulcers. J Am Acad Dermatol. 2003;48:207-214.

7. Kucia M, Machaliński B, Ratajczak MZ. The developmental deposition of epiblast/germ cell-line derived cells in various organs as a hypothetical explanation of stem cell plasticity? Acta Neurobiol Exp. 2006;66:331-341.

8. Kucia M,Zuba-Surma E, Wysoczyński M et al. Physiological and pathological consequences of identification of very small embryonic like (VSEL) stem cells in adult bone marrow. J Physiol Pharmacol. 2006;57:5-18.

9. Barthel R, Aberdam D. Epidermal stem cells. J Eur Acad Dermatol Venereol. 2005;19:405-413.

10. Lavker RM, Sun TT. Epidermal stem cells: properties, markers and location. Proc Natl Acad Sci. 2000;97:13473-13475.

11. Lehrer MS, Sun TT, Lavker RM. Strategies of epithelial repair: modulation of stem cell and transit amplifying cell proliferation. J Cell Sci. 1998;111:2867-2875.

12. Levy V, Lindon C, Zheng Y, Harfe BD, Morgan BA. Epidermal stem cells arise from the hair follicle after wounding. The FASEB J. 2007;21:1358-1366.

13. Linheng Li, Ting X. Stem cell niche: structure and function. Ann Rev Cell Dev Biol. 2005;21:605-631.

14. Taylor G, Lehrer MS, Jensen PJ, Sun TT, Lavker RM. Involvement of follicular stem cells in forming not only the follicle but also the epidermis. Cell. 2000;102:451-461.

15. Tumbar T, Guasch G, Greco V, Blanpain C, Lowry WE, Rendl M et al. Defining the epithelial stem cell niche in skin. Science. 2004:303:359-363.

16. Kucia M, Reca R, Campbell FR et al. A population of very small embryonic-like (VSEL) CXCR4(+)SSEA-1(+)Oct$4(+)$ stem cells identified in adult bone marrow. Leukemia. 2006;20:857-869.

17. RatajczakMZ, Machalinski B, Wojakowski W, Ratajczak J, Kucia M. A hypothesis for an embryonic origin of pluripotent Oct- $4^{+}$stem cells in adult bone marrow and other tissues. Leukemia. 2007;21:860-867.

18. Kucia M, Ratajczak J, Ratajczak MZ. Are bone marrow stem cells plastic or heterogeneous - that is the question? Exp Hematol. 2005;33:613-623.

19. Hałasa M, Baskiewicz-Masiuk M, Dabkowska E, Machalinski B. An efficient two-step method to purify very small embryonic-like (VSEL) stem cells from umbilical cord blood (UCB). Folia Histochem Cytobiol. 2008;2: 239-243.

20. Kucia M, Hałasa M, Wysoczynski M et al. Morphological and molecular characterization of novel population of CXCR4 (+) SSEA-4 (+) Oct-4 (+) very small embryoniclike stem cells purified from human cord blood - preliminary report. Leukemia. 2007;21:297-303.

21. Zuba-Surma EK, Kucia M, Klich I et al. Optimalization of isolation and further molecular and functional characterization of SSEA $-4^{+} / \mathrm{Oct}-4^{+} / \mathrm{CD} 133^{+} / \mathrm{CXCR} 4^{+} / \mathrm{LIN}-$ neg/CD45neg Very Small Embryonic-Like (VSELs) stem cells isolated from umbilical cord blood. Blood. 2008; 112:807.
22. Paczkowska E, Kucia M, Koziarska D et al. Clinical evidence that very small embryonic-like stem cells are mobilized into peripheral blood in patients after stroke. Stroke. 2009;40, 1237-1244.

23. Wojakowski W, Tendera M, Kucia M et al. Mobilization of bone marrow-derived Oct- $4^{+}$SSEA- $4^{+}$very small embryonic-like stem cells in patients with acute mycardial infarction. J Am Coll Cardiol. 2009;53:1-9.

24. Baumert B, Grymuła K, Pietruszka D et al. An optimalization of hematopoietic stem and progenitor cell isolation for scientific and clinical purposes by the application of a new parameter determining the hematopoietic graft efficacy. $\mathrm{Fo}$ lia Histochem Cytobiol. 2008;3:299-305.

25. Ratajczak MZ, Zuba-Surma E, Kucia M, Rece R, Wojakowski W, Ratajczak J. The pleotropic effect of the SDF-1-CXCR4 axis in organogenesis, regeneration and tumorigenesis. Leukemia. 2006;20:1915-1924.

26. Valentin G, Haas P, Gilmour D. The chemokine SDF-1a coordinates tissue migration through the spatially restricted activation of CXCR7 and CXCR4b. Curr Biol. 2007;17:1026-1031.

27. Poziomkowska-Gęsicka I, Słowik-Zyłka D, SłuczanowskaGłąbowska $\mathrm{S}$ et al. CXCR7-nowy receptor wiążący czynnik wzrostowy pochodzenia stromalnego (SDF-1). Post Biol Kom. 2010;37:553-570.

28. Yu H, Fang D, Kumar SM et al. Isolation of novel population of multipotent adult stem cells from human hair follicles. Am J Pathol. 2006;168:1879-1888.

29. Dyce PW, Wen L, Li J. In vitro germline potential of stem cells derived from fetal porcine skin. Nat Cell Biol. 2006;8:384-390.

30. Tani H, Morris RJ, Kaur P. Enrichment for murine keratinocyte stem cells based on cell surface phenotype. Proc Natl Acad Sci. 2000;97:10960-10965.

31. Nakamura Y, Muguruma Y, Yahata $T$ et al. Expression of CD90 on keratinocyte stem/progenitor cells. Br J Dermatol. 2006;154:1062-1070.

32. Drukała J, Majka M, Ratajczak M. Postępy w metodach izolacji i namnażania komórek macierzystych naskórka ludzkiego. Post Biol Kom. 2003;30:37-48.

33. Oshima H, Rochat A, Kedzia C, Kobayashi K, Barrandon Y. Morphogenesis and renewal of hair follicles from adult multipotent stem cells. Cell. 2001;104:233-245.

34. Drukala J, Majka M, Kwarciak A, Puchala J, Ratajczak MZ. Population of Rh123dim human keratinocytes form holoclones. Cent Eur J Biol. 2009;4:154-162.

35. Roh C, Lyle S. Cutaneous stem cells and wound healing. Pediatr Res. 2006;59:100R-103R.

36. Alonso L, Fuchs E. Stem cells of the skin epithelium. Proc Natl Acad Sci. 2003;100:11830-11835.

37. Fan Q, Yee CL, Ohyama M et al. Bone marrow-derived keratinocytes are not detected in normal skin and only rarely detected in wounded skin in two different murine models. Exp Hematol. 2006;34:672-679.

38. Borue $\mathrm{X}$, Lee $\mathrm{S}$, Grove $\mathrm{J}$ et al. Bone marrow-derived cells contribute to epithelial engraftment during wound healing. Am J Pathol. 2004;165:1767-1772.

39. Inokuma D, Abe R, Fujita Y et al. CTACK/CCL27 accelerates skin regeneration via accumulation of bone marrow-derived keratinocytes. Stem Cells. 2006;24:2810-2816.

40. Simka M. Delayed healing of chronic leg ulcers can result from impaired trafficking of bone marrow-derived precursors of keratinocytes to the skin. Med Hypotheses. 2007;69:637-641. 